\title{
Interprétation d'essai de pompage dynamique dans les enceintes fermées
}

\author{
Analysis of dynamic pumping test in a confined area
}

\author{
Y. IAGOLNITZER, A. MONNET \\ Entreprise BACHY*
}

Rev. Franç. Géotech, n55, pp. $34-45$ (avril 1991)

\section{Résumé}

La réalisation de bâtiments avec épuisement permanent à l'abri d'enceintes fermées nécessite une évaluation précise du débit d'exhaure résiduel, qu'aucune démarche classique ne permet actuellement de fournir.

Cet article décrit une méthode simple et rapide résolvant ce type de problème. Découlant d'une analyse hydraulique, la méthode proposée se traduit en pratique par un essai de pompage en deux temps : une période de pompage suivie d'une période de remontée.

Après une approche théorique de la méthode, un exemple pratique en illustre l'application.

Enfin, des précisions sont données sur la mise en œuvre de l'essai et son interprétation.

\section{Abstract}

Carrying out of buildings equiped with permanent pumping in a confined area has lead to the necessity of an accurate assessment of the residual discharge, that none of the classic approaches is currently able to work out.

This article describes a simple and fast method solving this type of problem. Derived from a hydraulic analysis, the method suggested here consists in practice of a two-time pumping test : a pumping period followed by a head recovery period.

After a theoretical approach to the method, a practical example illustrates its application.

Finally, precisions are given with regard to the setting up of the test and its interpretation. 
La reprise ces dernières années des investissements immobiliers a entraîné la multiplication des fouilles réalisées à l'abri d'« enceintes fermées » avec un pompage permanent.

Il s'agit de manière typique de fouilles urbaines destinées à recevoir en sous-sol des garages, des locaux techniques ou des magasins. Les surfaces varient de 1000 à $10000 \mathrm{~m}^{2}$ au sol. Le dallage du niveau inférieur est situé entre 5 et $15 \mathrm{~m}$ sous le niveau de la nappe phréatique. Ces conditions conduisent à prévoir des débits d'exhaure faibles, de 30 à $150 \mathrm{~m}^{3} /$ heure, qui permettent de justifier économiquement des solutions avec épuisement permanent. La paroi verticale de l'enceinte est réalisée en paroi moulée dans le sol. Le fond étanche peut être soit une couche géologique de faible perméabilité, soit un radier injecté.

Un moment critique de l'avancement du chantier est l'essai de pompage qui marque la fin de réalisation de l'enceinte, et permet d'entamer les terrassements au-dessous du niveau de la nappe. Moment critique, puisque le résultat de l'essai justifie ou infirme les choix de conception (radier injecté ou fiche de l'écran dans une couche étanche, ...) et sanctionne l'accomplissement des obligations contractuelles de l'entreprise spécialisée qui réalise l'enceinte.

Jusqu'à présent, les méthodes employées afin d'évaluer le débit résiduel à partir d'un essai de pompage en fin de réalisation d'une enceinte fermée relevaient des trois démarches suivantes :

1. considérations sur les volumes et les débits à partir d'une hypothèse sur le coefficient d'emmagasinement du terrain ;

2. considérations qualitatives lors du pompage d'essai en concluant que les exigences contractuelles sont satisfaites si le niveau d'eau dans la fouille continue à descendre pour un débit de pompage inférieur au débit imposé ;

3. évaluation du débit résiduel par un pompage à débit et niveaux stabilisés dans les conditions finales de rabattement.

Les méthodes 1 et 2 , souvent utilisées de façon complémentaire, ne permettent pas de conclure lorsque le débit résiduel est proche du débit contractuel. La première en effet ne donne du débit résiduel réel qu'une approximation très grossière et souvent erronée, les calculs étant basés sur des hypothèses non vérifiables concernant des coefficients par ailleurs assez mal connus ; la seconde est trop imprécise et hasardeuse.

La troisième méthode permet d'évaluer avec une bonne précision le débit résiduel, mais demande du temps. Elle nécessite environ une semaine dans des conditions favorables, cette durée augmentant en fonction de la profondeur du fond de fouille final et s'avérant presque toujours incompatible avec les délais impartis.

La méthode d'évaluation du débit résiduel présentée ici comporte une méthodologie d'essai et d'interprétation qui pallient les inconvénients décrits plus hauts: c'est une méthode quantitative de courte durée basée sur la loi de conservation et ne faisant appel à aucune hypothèse particulière sur la couche de terrain concernée.
Le chapitre 1 présente la modélisation du problème et la description d'une méthodologie d'essai.

Le chapitre 2 illustre la mise en application de cette méthode par l'étude détaillée d'un exemple concret.

Le chapitre 3 est une discussion portant sur des points particuliers de la procédure d'essai, de l'analyse des mesures et de l'interprétation.

\section{MODÉLISATION DU POMPAGE}

\subsection{Schéma de principe}

Le schéma de la figure 1 présente le cas classique de la mise hors d'eau d'une fouille protégée par une paroi moulée. Le fond de l'enceinte, représenté ici par un radier injecté, peut être remplacé de façon équivalente par le recoupement de la paroi sur un horizon peu perméable.

\subsection{Loi de conservation et expression des différents débits}

La loi de conservation de la masse nous permet d'écrire une équation liant les débits : la somme des débits entrants dans la fouille est égale à la somme des débits sortants.

- Débit entrant et assimilé :

$\mathrm{Q} e$, débit résiduel traversant l'enceinte, il représente le débit par le fond grossi du débit latéral. Avec une paroi moulée périmétrale, ce débit latéral est généralement négligeable (par contre dans le cas d'un écran vertical injecté il y aurait lieu d'en tenir compte).

Qs, débit d'essorage du terrain à l'intérieur de la fouille.

- Débit sortant :

Qp, débit pompé.

L'équation de base s'exprime donc simplement par:

$$
\mathrm{Qp}=\mathrm{Qs}+\mathrm{Qe}^{\mathrm{p}}
$$

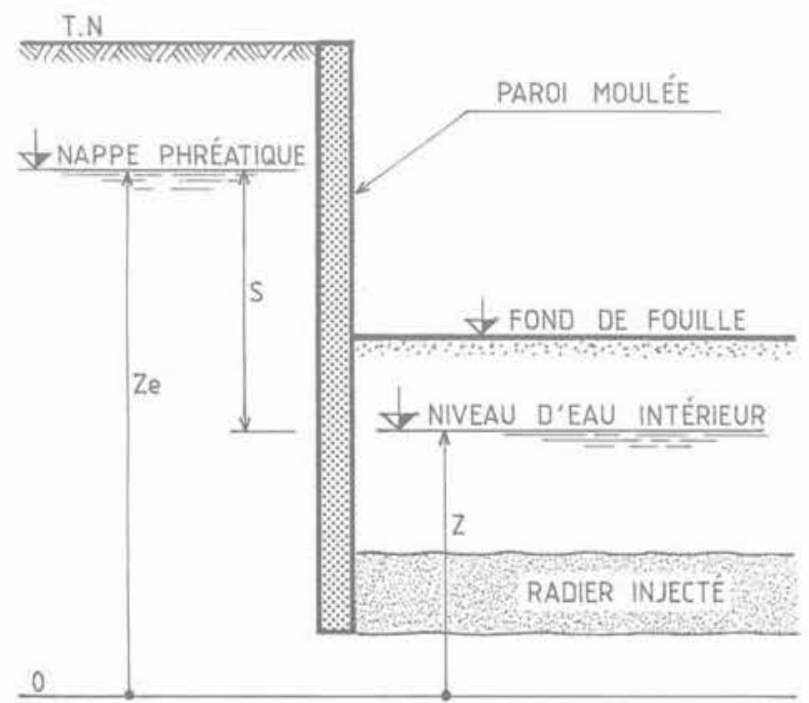

Fig. 1. - Schéma de principe.

Fig. 1. - General layout. 


\subsubsection{Débit entrant et transmissivité}

Le débit entrant dans la fouille, Qe, encore appelé débit résiduel ou débit de fuite, constitue l'inconnue du problème.

Cependant cette inconnue n'est pas une constante : elle dépend de la différence de charge s entre la nappe extérieure et le niveau d'eau à l'intérieur de la foulle. On admet la relation de proportionnalité suivante :

$$
\mathrm{Qe}=\mathrm{T} \cdot \mathrm{s}
$$

où $T$, transmissivité globale de la fouille, représente le débit que peut fournir cette fouille par mètre de rabattement; elle sera exprimée par la suite de $\mathrm{m}^{3} / \mathrm{h}$ par $\mathrm{m}$ de rabattement, que l'on préfère à la notation usuelle $\left(\mathrm{m}^{2} / \mathrm{s}\right)$.

Indépendante du niveau d'eau dans la fouille, et de tout autre paramètre. T est en théorie une constante. On verra en 1.3. la manière de l'évaluer par le calcul, et l'explication des variations qui sont observées dans la réalité.

Il est important de faire ici la distinction entre la transmissivité globale définie ci-dessus et le « débit spécifique "utilisé dans nombre d'essais de pompage, et qui représente communément le rapport entre un débit pompé (en période de pompage, donc), et un rabattement $(\mathrm{Qp} / \mathrm{s})$. C'est une entité variable au cours du temps et sans intérêt dans le cadre de la présente méthode. Une comparaison entre transmissivité globale et débit spécifique sera menée en 3.3.1.

\subsubsection{Débit d'essorage}

Pendant un intervalle de temps $\mathrm{dt}$, le niveau intérieur 2 varie de $\mathrm{dz}$, et le débit d'essorage Qs, proportionnel à la surface $S$ de la fouille, s'exprime par:

$$
\mathrm{Qs}=\gamma \cdot \mathrm{S} \cdot \frac{\mathrm{ds}}{\mathrm{dt}}
$$

Le coefficient de proportionnalité est le classique (et insaisissable !) coefficient d'emmagasinement, exprimé en $\%$.

En régime permanent, il est aussi nommé porosité efficace, $\mathrm{n}_{\mathrm{e}}$ (rapport du volume d'eau libre sur le volume de sol).

La plupart des analyses d'essais de pompage était jusqu'à présent basée sur une hypothèse sur le coefficient d'emmagasinement, permettant des calculs simples mais très approximatifs : une des inconnues étant éliminée, le problème posé se réduisait à une simple équation à une inconnue : Qe... Mais aucune justification sérieuse de débit résiduel ne pouvait en être tirée.

Il est clair que le débit d'essorage est lié au caractère dynamique du niveau de l'eau dans la fouille: à niveau stabilisé, ce terme est nul et l'équation des débits se réduit à $\mathrm{Qe}=\mathrm{Qp}$.

\subsubsection{Débit de pompage}

Le débit extrait de la fouille est noté $\mathrm{Qp}$ et est mesuré sur le chantier à l'aide de compteurs (on verra plus tard l'importance de cet équipement).

\subsection{Résolution de l'équation}

En remplaçant les expressions (2) et (3) de débits développées plus haut dans l'équation (1), on obtient ;

$$
\mathrm{Qp}=\mathrm{T} \cdot\left(\mathrm{z}_{\mathrm{e}}-\mathrm{z}\right)+\gamma \cdot \mathrm{S} \cdot \frac{\mathrm{ds}}{\mathrm{dt}}
$$

ou encore :

$$
z+\gamma \cdot \frac{S}{T} \cdot \frac{d z}{d t}=z_{e}-\frac{Q p}{T}
$$

Précisons que dans cette équation, les seules valeurs inconnues sont $\gamma$ et $T$, les autres $\left(z, z_{e}, t, Q p\right.$ et $\left.S\right)$ étant connues ou mesurées durant l'essai.

Pour simplifier la manipulation des données, on introduit une grandeur physique nouvelle;

$$
\tau=\frac{\gamma \mathrm{S}}{\mathrm{T}}
$$

qui a la dimension d'une durée. Elle sera nommée par la suite «temps de relaxation de la fouille ».

L'équation différentielle (4) s'exprime donc simplement par :

$$
\mathrm{z}+\tau \frac{\mathrm{dz}}{\mathrm{dt}}=\mathrm{z}_{e} \frac{\mathrm{Qp}}{\mathrm{T}}
$$

Dans la plupart des cas, le niveau de la nappe phréatique est très peu variable (quelques centimètres tout au plus). On peut alors, en prenant comme hypothèse la stabilité de la nappe $(\mathrm{ds}=-\mathrm{dz})$, écrire léquation précédente en fonction du rabattement s:

$$
s+\tau \frac{\mathrm{ds}}{\mathrm{dt}}=\frac{\mathrm{Qp}}{\mathrm{T}}
$$

Dans les deux cas particuliers suivants, les solutions de l'équation sont connues :

- Cas où Qp est nul (pas de pompage)

L'équation de conservation des débits.

$\mathrm{s}+\tau \mathrm{ds} / \mathrm{dt}=0$ a alors pour solution :

$$
s=s_{0} \cdot e^{-t / \tau}
$$

Si à un instant donné, alors qu'un rabattement $\mathrm{s}_{0} \mathrm{a}$ été obtenu, le pompage est arrêté, le niveau remonte suivant une loi d'amortissement exponentiel.

Ou encore, sur une courbe de remontée après arrêt de pompage, il est possible de calculer, sur l'intervalle entre deux mesures, un temps de relaxation :

$$
\tau=\frac{\mathrm{dt}}{\operatorname{Ln} \frac{\mathrm{so}}{\mathrm{s}}}
$$

où : $\mathrm{dt}$ est la durée de l'intervalle entre deux mesures, $\mathrm{s}_{0}$ le rabattement à l'origine de l'intervalle, $\mathrm{s}$ le rabattement en fin d'intervalle. 
On comprend ici le contenu physique pratique du temps de relaxation: tout pompage arrêté, c'est le temps nécessaire pour obtenir un rabattement $s$ tel que $\operatorname{Ln}\left(\mathrm{s}_{0} / \mathrm{s}\right)=1$, ou encore $\mathrm{s}=\mathrm{s}_{0} / e$, c'est-à-dire un rabattement résiduel s ramené sensiblement au tiers du rabattement initial $s_{0}$.

- Cas où $\mathrm{Qp}$ est constant

L'expression de la solution $s$ est alors :

$$
S=s_{0} \cdot e^{-t / \tau}+\frac{Q p}{T} \cdot\left(1-e^{-t / T}\right)
$$

Il est donc possible, sur chaque intervalle de mesure en période de pompage, de faire une estimation de la transmissivité de la fouille:

$$
T=Q p \cdot \frac{1-e^{-1 / \pi}}{s-s_{0} \cdot e^{-t / T}}
$$

pour peu que l'on dispose d'une estimation du temps de relaxation sur la tranche de terrain considérée par l'interprétation précédente. On peut alors inférer le débit résiduel de la fouille pour un rabattement donné $\mathrm{s}$, en écrivant que $\mathrm{Qe}=\mathrm{T}, \mathrm{s}$.

\subsection{Principe de l'essai de pompage}

Comme pour tout autre essai de pompage, il faut prévoir la mise en place d'un dispositif permettant de réaliser le pompage et de mesurer les éléments suivants :

- quantités d'eau pompée, à l'aide de compteurs volumétriques ou de débitmètres enregistreurs ;

- niveau de la nappe extérieure, par une série de piézomètres extérieurs ;

- niveau d'eau à lintérieur de la fouille, par une série de piézomètres intérieurs.

Il est, de plus, important de tenir compte du temps d'installation de ce dispositif dans le planning, car étant de l'ordre d'une semaine, il est plus long que l'essai lui-même.

\section{Méthodologie de l'essai}

Reprenant les deux phases de calcul du chapitre précédent, le déroulement de l'essai comportera deux étapes ;

- une période à débit nul, ou période de remontée ;

- une période à débit de pompage constant.

Cependant, si les calculs doivent être effectués dans cet ordre précis, lessai sera en pratique réalisé dans lordre inverse: la période de remontée nécessitant un rabattement initial, c'est la période de pompage qui marquera la première phase de l'essai, après un pompage préliminaire destiné à s'assurer du bon fonctionnement de tous les éléments du dispositif.

a. Une période de pompage à « débit constant ».

On considérera que le débit reste constant pour chaque intervalle de temps, sur lequel un débit moyen sera calculé.

Les mesures effectuées durant cette période permettront de calculer la transmissivité globale de la fouille $\mathrm{T}$ sur chaque intervalle de temps (cf. équation 6), au moyen de la valeur de $\tau$ déduite de la période de remontée.

b. Une période de remontée $(\mathrm{Qp}=0$; arrêt du pompage).

Les mesures faites durant cette période permettront de calculer le temps de relaxation de la fouille $\tau$ (cf. équation 5). L'obtention d'un rabattement initial significatif est nécessaire pour débuter la remontée. Un minimum de $1,50 \mathrm{~m}$ a été utilisé jusqu'à présent. D'autre part, un minimum de $8 \mathrm{~h}$ pour la durée de la remontée a toujours été respecté ; il est cependant intéressant de poursuivre les mesures au-delà, lorsque les conditions de chantier le permettent.

Avant le début de l'essai, il est bon de relever les niveaux des piézomètres extérieurs afin d'appréhender les éventuelles fluctuations de la nappe. D'autre part, dès le début de l'essai, des mesures complètes comprenant les relevés des compteurs volumétriques et des piézomètres extérieurs et intérieurs, doivent être prises à intervalles de temps réguliers. Deux heures est l'intervalle qui a été utilisé jusqu'à présent, un intervalle plus court ne permettant pas d'améliorer la précision des résultats d'une manière significative.

Ainsi que le montre l'ensemble du chapitre suivant, l'évaluation du débit résiduel permet ensuite le calcul de la perméabilité $K$ du radier et du coefficient d'emmagasinement du terrain $\gamma$.

\section{APPLICATION}

La méthode qui vient d'être décrite a permis l'interprétation de plusieurs essais de pompage dans la région parisienne:

- Zac du Front de Seine, Levallois

- llot 2-1: pompage du 11/09/89;

- llot 2-6: pompage du 07/08/90;

- Axe-Seine, rue Rouget-de-I'Isle, Issy-les-Moulineaux :

- bâtiment D : pompage du 29/01/90;

- bâtiment E.F.G. : pompage du 09/05/90 ;

- Aérospatiale, Zac Louis-Blériot, Suresnes;

- bâtiment Su1 : pompage du 03/04/90 ;

- Henripré, face 120 , rue Galiéni, Boulogne-Billancourt pompage du 20/01/90;

- nouveau siège TF1, quai du Point du jour, Boulogne ;

- zone IGH : pompage du 29/03/90;

- zone Activité : pompage du 11/05/90.

Les radiers de ces chantiers ont une caractéristique commune : ils sont injectés dans la craie. Cependant, l'épaisseur de cette dernière au-dessus du radier, sa nature ainsi que celle des couches géologiques supérieures (alluvions anciennes ou modernes), diffèrent suivant les chantiers.

L'exemple de description et d'interprétation présenté ci-dessous correspond à l'essai de pompage réalisé sur le chantier d'Axe-Seine, bâtiment D.

L'exploitation des mesures (graphiques et calculs) a été réalisée au moyen du tableur du logiciel WORKS. 


\subsection{But de l'essai}

Dans le cadre de la réalisation d'une paroi moulée périphérique et d'un radier injecté dans la craie entre les cotes 13,50 Ngf et 16,50 Ngf, l'essai de pompage permet d'évaluer le débit résiduel dans la fouille pour une nappe phréatique à $27 \mathrm{Ngf}$ et un rabattement de $5 \mathrm{~m}$ dans la fouille.

\subsection{Dispositif de pompage}

Le dispositif de pompage mis en place comporte;

- trois puits Pu1, Pu2 et Pu3, crépinés sur une hauteur de $5,40 \mathrm{~m}$.

Pul a une profondeur de $8,90 \mathrm{~m}$ et est équipé d'une pompe Grundfos de débit nominal $80 \mathrm{~m}^{3} / \mathrm{h}$;

Pu2 a une profondeur de 9,20 m et est équipé d'une pompe KSB de débit nominal $20 \mathrm{~m}^{3} / \mathrm{h}$;

Pu3 a une profondeur de $9,10 \mathrm{~m}$ et est équipé d'une pompe KSB de débit nominal $10 \mathrm{~m}^{3} / \mathrm{h}$;

- trois piézomètres extérieurs $\mathrm{P}_{21}, \mathrm{P}_{22}, \mathrm{P}_{23}$ dont les prises de pression sont situées dans les alluvions anciennes (approximativement $18 \mathrm{Ngf}$ ) .

- dix piézomètres intérieurs $\mathrm{P}_{24}$ à $\mathrm{P}_{213}$, ancrés à une cote moyenne de $18 \mathrm{Ngf}$;

- deux compteurs volumétriques, l'un installé sur la pompe de plus gros débit, l'autre sur une canalisation recueillant les eaux des deux autres puits.

Les figures 2 et 3 montrent la disposition géographique des éléments décrits ci-dessus.

\subsection{Procédure de l'essai}

\subsubsection{Mise en route et préparation}

Les pompages ont débuté le 29 janvier 1990 à $16 \mathrm{~h}$ avec la mise en route simultanée des trois pompes.

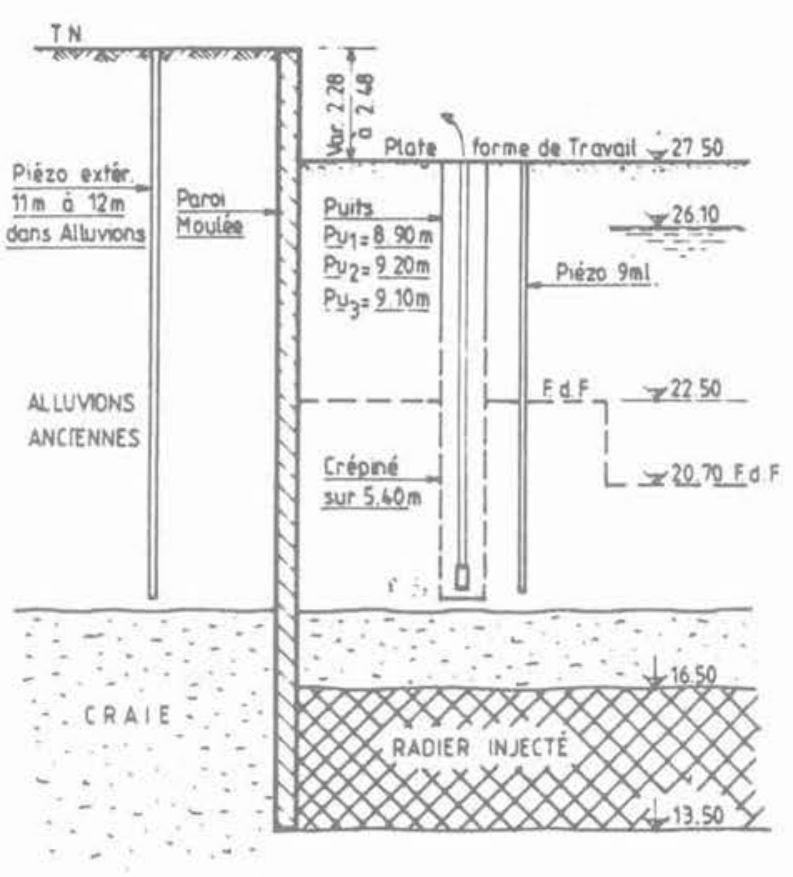

Fig. 3. - Coupe sur dispositif de pompage.

Fig. 3. - Section of the pumping system.

Des relevés périodiques des niveaux dans tous les piézomètres ont été pris régulièrement. Le débit pompé, de l'ordre de $90 \mathrm{~m}^{3} / \mathrm{h}$ au départ fut baissé progressivement.

\subsubsection{L'essai de pompage}

- Période de pompage :

Le 30 janvier à 11 h 15 , le rabattement avait atteint $3,3 \mathrm{~m}$. Les compteurs volumétriques ont été installés

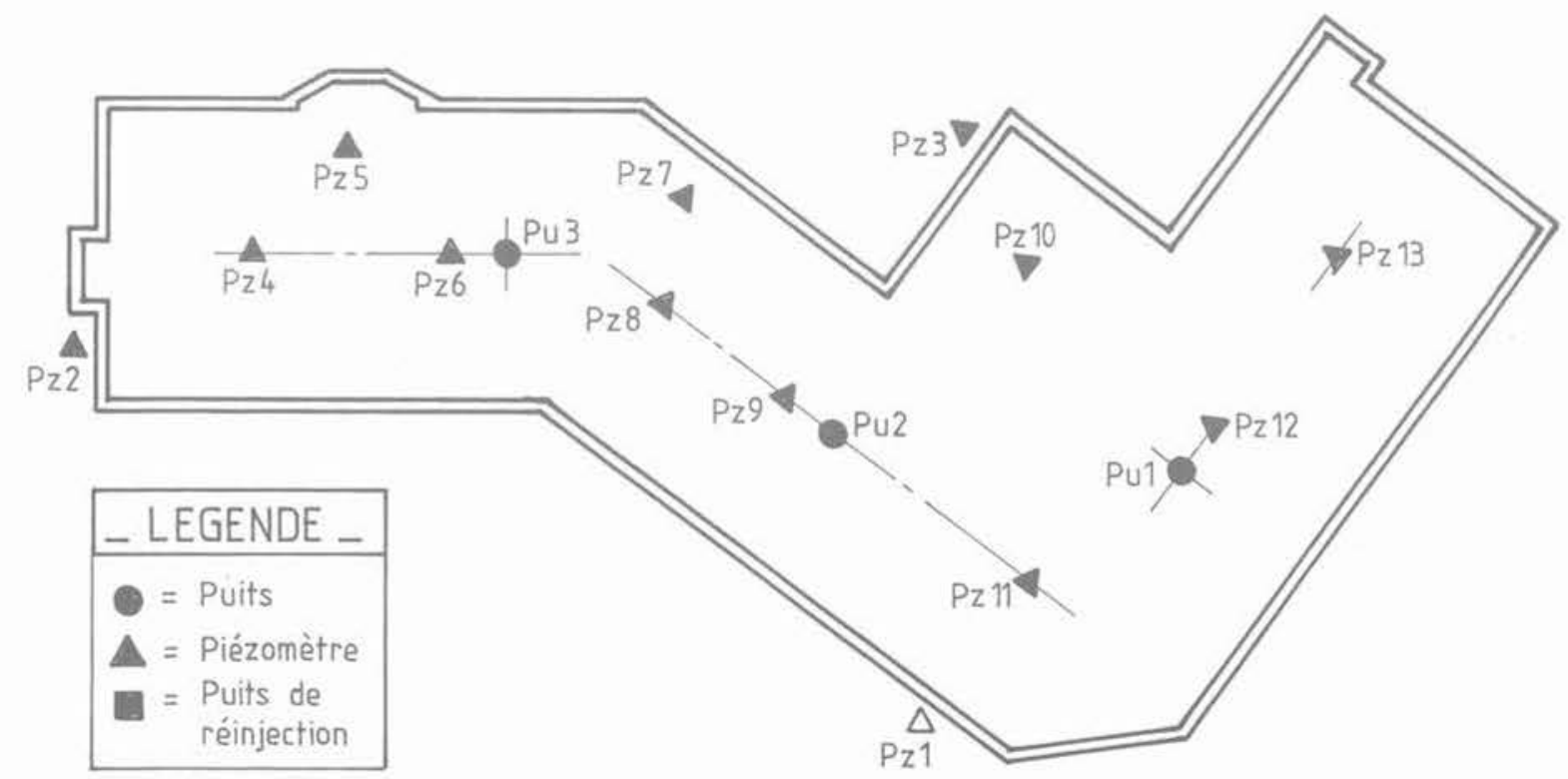

Fig. 2. - Implantation des puits de pompage et des piézomètres.

Fig. 2. - Layout of pumping wells and piezometers. 
et tous les relevés pris régulièrement à intervalle de $2 \mathrm{~h}$, sur une durée de $8 \mathrm{~h}$;

- Période de remontée :

le pompage a été arrêté le 30 janvier à 19 h 20 et les mesures des niveaux prises toutes les $2 \mathrm{~h}$ sur une durée de $12 \mathrm{~h}$. L'essai s'est terminé le 31/01/90 à 7 h 20 .

Deux incidents sont à signaler :

- un arrêt des pompes de quelques minutes, nécessaire pour permettre la mise en place des compteurs volumétriques, ayant eu lieu avant le début de l'essai, et donc sans conséquence sur celui-ci ;

- une faible ondée dans la nuit du 30 au 31 janvier, qui ne sera pas prise en compte.

\subsubsection{Consignation et représentation des mesures}

Tous les relevés des piézomètres intérieurs depuis la mise en route de l'essai sont présentés graphiquement (fig. 4 et 5 ).

\subsection{Analyse des mesures}

\subsubsection{Niveaux extérieurs}

Les niveaux extérieurs sont stables durant l'essai. Les piézomètres $\mathrm{Pz} 1$ et $\mathrm{Pz} 2$ sont stabilisés à $26,10 \mathrm{Ngf}$, tandis que le piézomètre $\mathrm{Pz} 3$ l'est à la cote légèrement inférieure de 25,95 Ngf. La différence entre ces deux niveaux n'excède pas $15 \mathrm{~cm}$ et peut être expliquée par la position de la fouille. L'une de ses faces est en effet séparée de la Seine par des fouilles adjacentes de profondeur similaire, formant écran. Le piézomètre $\mathrm{Pz} 3$, installé dans cette zone, contrairement aux autres piézomètres extérieurs, indique donc une charge d'eau légèrement inférieure.

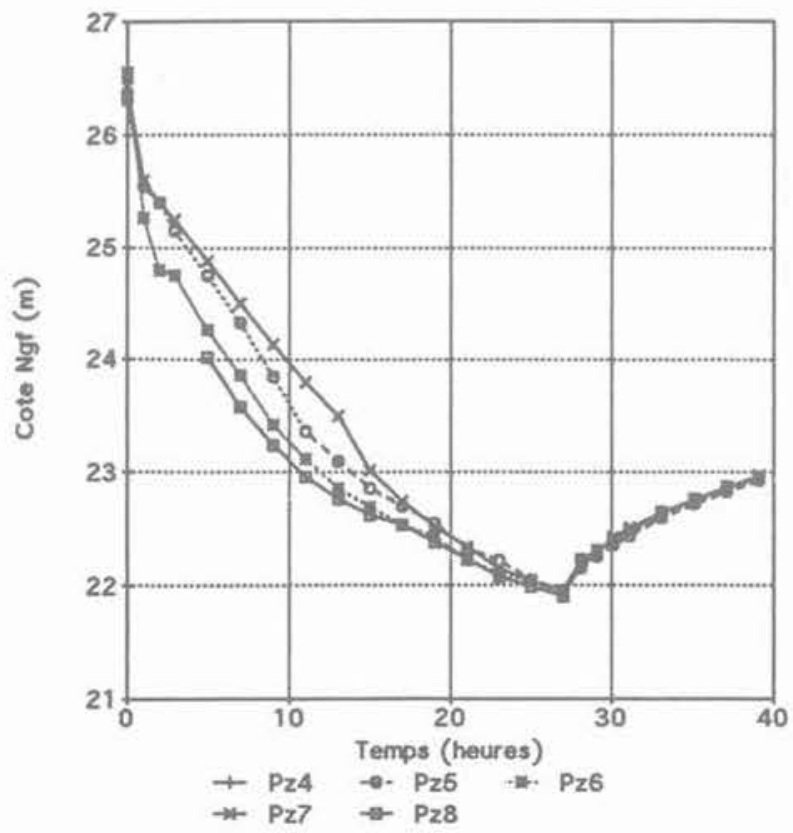

Fig. 4. - Niveau des piézomètres intérieurs Pz4 à Pz8. Fig. 4. - Piezometric head of inside piezometers Pz4 to Pz8.

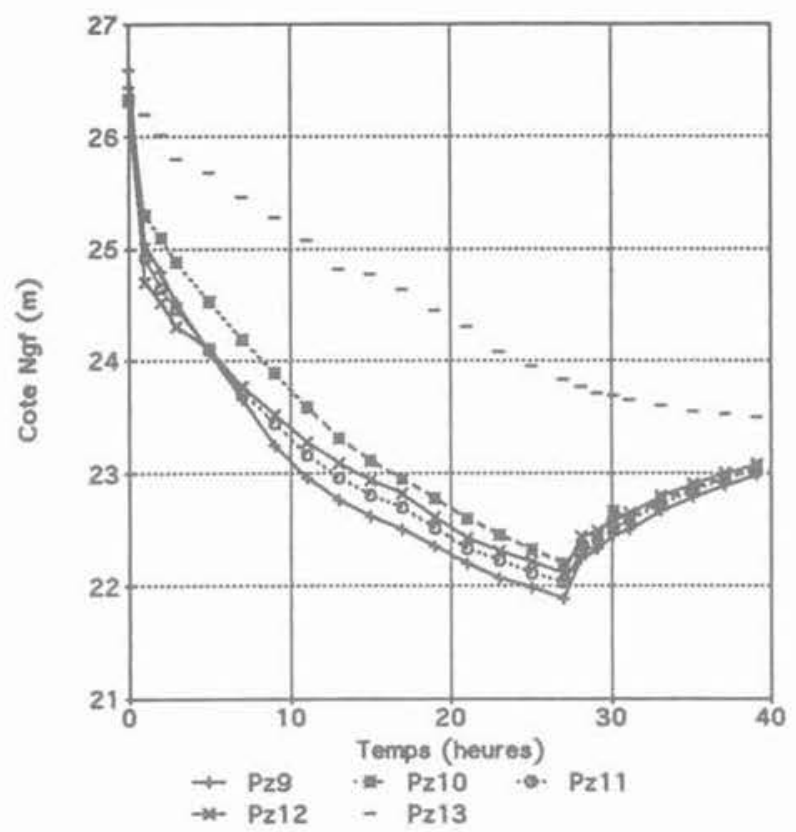

Fig. 5. - Niveau des piézomètres intérieurs $P_{29}$ à $P_{z} 13$. Fig. 5. - Piezometric head of inside piezometers Pz9 to Pz13.

Pour les calculs, les niveaux moyens extérieurs seront pris égaux à la moyenne de Pz3 et de la moyenne de $\mathrm{Pz} 1$ et $\mathrm{Pz} 2$. Certaines valeurs sont manquantes, mais une interpolation linéaire parait raisonnable.

\subsubsection{Niveaux intérieurs}

Le piézomètre $\mathrm{P}_{2} 13$ réagit avec un très grand retard ; son niveau continue en effet à descendre pendant la remontée. Il ne sera pas pris en compte dans les calculs. Les autres piézomètres présentent en outre des courbes de rabattement très proches. Les calculs seront menés à partir d'un niveau piézométrique intérieur moyen pris égal à la moyenne des niveaux des piézomètres $\mathrm{Pz} 4$ à $\mathrm{Pz} 12$.

\subsubsection{Calculs de débits}

Les débits moyens, calculés sur chaque intervalle de temps à partir des relevés des compteurs volumétriques, s'établissent comme suit :

- entre $13 \mathrm{~h} 15$ et 15 h $15: 38,6 \mathrm{~m}^{3} / \mathrm{h}$;

- entre 15 h 15 et 17 h $15 ; 33,5 \mathrm{~m}^{3} / \mathrm{h}$;

- entre 17 h 15 et 19 h $15: 27,6 \mathrm{~m}^{3} / \mathrm{h}$.

\subsection{Interprétation}

L'interprétation des résultats peut être décomposée en différentes phases : recherche d'un temps de relaxation puis d'une transmissivité réalistes, et déduction du débit résiduel, du coefficient d'emmagasinement du terrain et de la perméabilité du radier.

\subsubsection{Calcul du temps de relaxation $\tau$}

Les calculs de $\tau$ sont menés pendant la période de remontée sur chaque intervalle de temps et la figure 6 montre son évolution en fonction du temps. La dernière valeur obtenue durant l'essai, la plus réaliste (voir paragraphe 3.3.1.), sera utilisée dans la suite des calculs: le temps de relaxation $\tau=65 \mathrm{~h}$. 


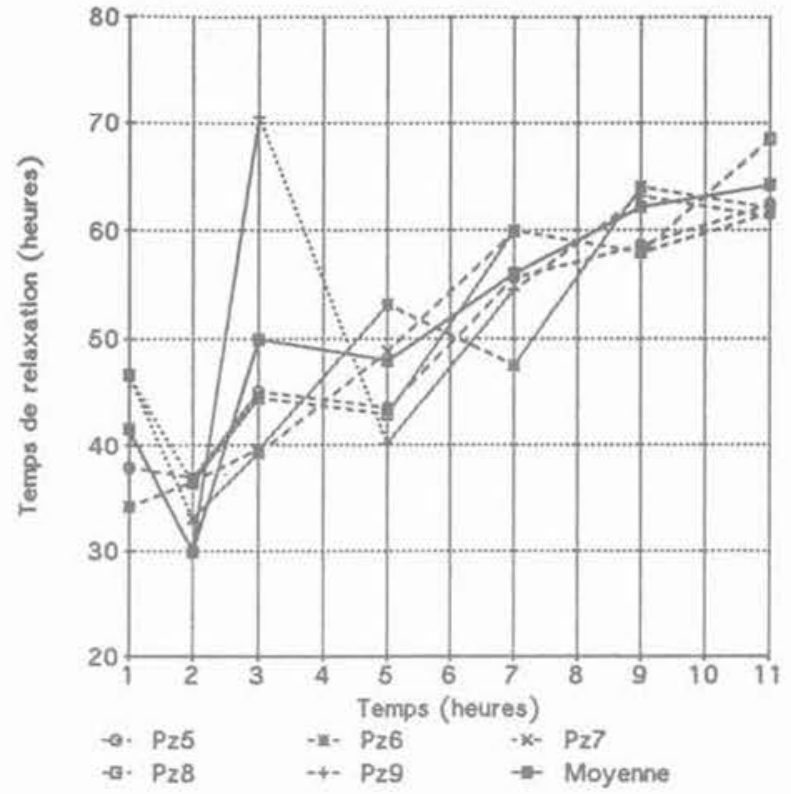

Fig. 6. - Evolution du temps de relaxation sur la période de remontée.

Fig. 6. - Evolution of the relaxation time over the head recovery period.

\subsubsection{Calcul de la transmissivité $T$}

T est calculé à partir des mesures faites lors de la période de pompage. Dans le cas présent, trois intervalles de temps ayant chacun une mesure de débit moyen permettent trois calculs de $\mathrm{T}$.

On trouve, dans lordre chronologique et à $2 \mathrm{~h}$ d'intervalle, les valeurs suivantes:

$$
\begin{aligned}
& T=6,12 \mathrm{~m}^{3} / \mathrm{h} / \mathrm{m} \\
& T=4,28 \mathrm{~m}^{3} / \mathrm{h} / \mathrm{m} \\
& T=3,93 \mathrm{~m}^{3} / \mathrm{h} / \mathrm{m}
\end{aligned}
$$

Les deux dernières valeurs sont du même ordre (différence de 0,35 ), et nettement inférieures à la première (différence moyenne de 2,02).

Or au début de l'essai, les pompes sont arrêtées afin d'installer les compteurs. Il s'opère alors un début de remontée; le régime permanent du pompage est interrompu. Le niveau de la nappe remonte légèrement, mais en raison du temps de retard des piézomètres et de la reprise assez rapide du pompage, cette hausse n'est pas enregistrée.

$\mathrm{A} \mathrm{t}=0, \mathrm{~s}_{0}$ est donc inférieur au $\mathrm{s}_{0}$ mesuré, et par conséquent (voir équation 6), le T déduit est supé. rieur au $\mathrm{T}$ réel.

Pour l'évaluation de $\mathrm{T}$, la première valeur est ignorée et la moyenne des deux autres donne une transmissivité de $4,1 \mathrm{~m}^{3} / \mathrm{h} / \mathrm{m}$.

\subsubsection{Calcul du débit résiduel $\mathrm{Qr}$}

En prenant $T=4,1 \mathrm{~m}^{3} / \mathrm{h} / \mathrm{m}$, on trouve pour une nappe extérieure à $27 \mathrm{Ngf}$ et un rabattement de $5 \mathrm{~m}$ une valeur de débit résiduel de $20,5 \mathrm{~m}^{3} / \mathrm{h}$.
2.5.4. Calcul du coefficient d'emmagasinement $\gamma$ Les calculs sont poursuivis avec la même valeur de T. $\gamma$ est donné par la relation :

$$
\gamma=\frac{\mathrm{T}}{\mathrm{S}} \cdot \tau
$$

et l'on peut tracer la courbe de l'évolution de $\gamma$ en fonction de la remontée de la nappe (voir fig. 7). Le coefficient $\gamma$ semble tendre vers une valeur voisine de $16 \%$

2.5.5. Calcul de la perméabilité $K$ du radier D'après la loi de Darcy,

$$
\mathrm{Qr}=\mathrm{K} \cdot \mathrm{S} \cdot \mathrm{i}=\mathrm{K} \cdot \mathrm{S} \cdot \frac{\mathrm{dH}}{\mathrm{ds}}
$$

soit :

$$
\mathrm{K}=\frac{\mathrm{Qr}}{\mathrm{S}} \cdot \frac{\mathrm{ds}}{\mathrm{dH}}
$$

Dans le cas présent :

$\mathrm{Qr}=20,5 \mathrm{~m}^{3} / \mathrm{h}$;

$\mathrm{S}=1670 \mathrm{~m}^{2}$;

$\mathrm{ds}=$ épaisseur $\mathrm{du}$ radier $=3 \mathrm{~m}$ :

$\mathrm{dH}=5 \mathrm{~m}$ :

d'où une perméabilité $\mathrm{K}$ de $2 \cdot 10^{-6} \mathrm{~m} / \mathrm{s}$.

\section{DISCUSSION}

Une analyse plus approfondie de l'essai de pompage réalisé sur le chantier d'Axe-Seine, ainsi que des autres essais effectués permet de définir d'une manière générale les conditions dans lesquelles doit se dérouler un essai de pompage et de mieux cerner la méthode d'interprétation des mesures.

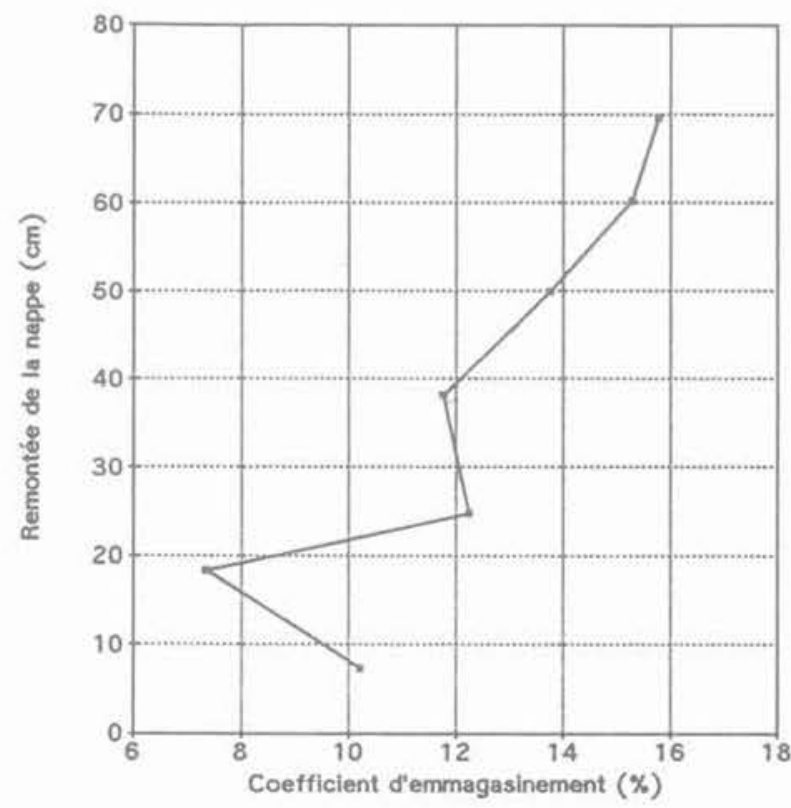

Fig. 7. - Evolution du coefficient d'emmagasinement en fonction de la remontée.

Fig. 7. - Evolution of the storage coefficient versus head recovery 


\subsection{Le dispositif de pompage}

\subsubsection{Nombre de puits}

Le nombre de puits à mettre en ceuvre dépend de la nature des couches rencontrées et de la surface de la fouille, ainsi bien sûr que des délais impartis. Par exemple, pour une même surface on prévoira d'autant plus de puits, toutes choses égales par ailleurs, que le terrain sera moins perméable.

SICHARDT, sur des considérations de gradient hydraulique maximum liées au risque de désorganisation du terrain lors du pompage, a établi une formule permettant d'évaluer le débit maximal d'un puits [2] :

$$
\mathrm{Q}=2 \cdot \pi \cdot \mathrm{r} \cdot \mathrm{h} \frac{\sqrt{\mathrm{K}}}{15}
$$

où : $r=$ rayon du forage $(m)$;

$\mathrm{h}=$ hauteur mouillée $(\mathrm{m})$;

$\mathrm{K}=$ perméabilité du terrain dans lequel le puits est crépiné $(\mathrm{m} / \mathrm{s})$.

D'autre part, en fonction d'un nombre de puits prévu, (et donc d'une capacité globale de pompage), de certaines hypothèses sur le coefficient d'emmagasinement, et en prenant pour transmissivité la transmissivité objectif du point de vue contractuel, on peut calculer au moyen de l'équation (6) le temps nécessaire pour obtenir un rabattement donné. Ainsi, en fonction des délais prévus pour l'essai de pompage, on peut estimer le nombre optimum de pompes. Cependant, cette prévision ne donne de résultats fiables que lorsque le terrain est déjà connu, et en particulier lorsqu'on connait son coefficient d'emmagasinement à court terme.

\subsubsection{Nombre de piézomètres et disposition}

L'expérience montre que 1 à 2 piézomètres par puits sont suffisants.

Il est conseillé de les disposer le plus loin possible des puits, afin d'éviter de se trouver dans leur cône de rabattement, et donc de relever un niveau d'eau nettement inférieur au niveau réel de l'eau dans la fouille.

\subsection{La procédure de l'essai}

\section{Les relevés}

Procéder au relevé de tous les piézomètres et des compteurs des puits nécessite environ $15 \mathrm{~min}$ (pour un équipement similaire à celui décrit dans l'exemple présenté). Or l'intervalle de temps entre deux relevés est de $2 \mathrm{~h}$, voire parfois 1 . On comprend alors qu'il est important que ces relevés soient effectués toujours dans le même ordre, sous peine d'obtenir des allures de rabattement irrégulières et des écarts de débit. Une autre possibilité consiste à relever l'heure de chaque mesure, mais elle rend la production des graphiques et les calculs d'interprétation pénibles.

\subsubsection{L'évaluation des débits}

Elle doit être la plus précise possible, car c'est elle qui conditionne la validité de l'interprétation.

Une bonne évaluation des débits peut se faire, par exemple, à laide de compteurs volumétriques, qui permettent de calculer un débit moyen sur l'intervalle de temps entre deux mesures. Les mesures instantanées de débit (ex : par chronométrage du remplissage d'une cuve) sont à éviter, sauf si elles ont un rôle complémentaire, car elles ne sont pas toujours représentatives du débit pompé réel, du moins pas à la précision recherchée. En particulier, elles n'intègrent pas les arrêts accidentels de pompage.

L'estimation de la transmissivité T est directement proportionnelle à $\mathrm{Qp}$ pour un intervalle de temps t durant la période de pompage. Rappelons ici l'équation (6) ;

$$
T=\frac{Q p}{s} \cdot \frac{1-e^{-t / \tau}}{1-\frac{s_{0}}{s} \cdot e^{-t / \tau}}
$$

En conséquence, quelle que soit la finesse de l'analyse, la précision de l'estimation de la transmissivité sera directement fonction de la précision des mesures de débit.

\subsubsection{Les incidents}

Tous les incidents augmentent la difficulté d'interprétation, et c'est pourquoi les premières heures de pompage doivent être consacrées à vérifier le bon fonctionnement des puits, des compteurs et des piézomètres (réglage des débits de pompe pour éviter la mise à sec des puits, soufflage des piézomètres bouchés....).

Cependant, comme il n'est pas possible de se protéger contre tous les aléas, il importe de noter en cours d'essai les facteurs susceptibles d'influencer son déroulement :

- arrêts éventuels de pompes (début et fin)

- orages, avec relevé de la hauteur d'eau tombée,...

\subsection{L'interprétation}

Dans un premier temps, des précisions seront apportées sur les valeurs du temps de relaxation et de la transmissivité, calculées dans les deux phases de calcul correspondant, la première à la période de remontée, la seconde à la période de pompage.

Par la suite, des considérations complémentaires sur le coefficient d'emmagasinement et le temps de retard des piézomètres permettront d'apporter des explications à certains phénomènes observés et d'affiner l'interprétation.

\subsubsection{Le temps de relaxation}

Les courbes de l'évolution du temps de relaxation pendant la période de remontée présentent deux particularités : 
a. des variations d'amplitudes variables à court terme (2 à $4 \mathrm{~h}$ ). Ces variations sont expliquées plus loin comme étant une conséquence des temps de retard ;

b. une croissance régulière vers une valeur limite.

Cette allure s'explique par la courbe de saturation du sol au-dessus du niveau d'eau dans la fouille, dont l'évolution pendant une période de régime transitoire est représentée sur la figure 8 .

- En début de remontée, le terrain situé au-dessus du niveau de l'eau dans la fouille est encore très saturé : le degré de saturation varie entre le degré de saturation * au repos " du sol hors eau et $100 \%$. Le coefficient d'emmagasinement $\gamma$ est faible, le temps de relaxation est donc sous-estimé.

- Au fur et à mesure de la remontée, le terrain supérieur au niveau d'eau s'essore de plus en plus et le coefficient d'emmagasinement $\gamma$ augmente, ainsi que $\tau$, qui tend vers sa limite.

Notons que le degré de saturation « au repos $»$ n'est pas nul et dépend de la proportion d'eau liée dans le terrain considéré.

Les calculs de $\tau$ sont menés par intervalles de temps successifs, ce qui permet d'éviter l'influence des variations de début de remontée qui auraient pour effet d'en abaisser artificiellement la valeur.

De plus, la valeur de $\tau$ la plus réaliste, et que l'on utilise dans les calculs, est la dernière de l'essai puisque l'on s'approche le plus d'un régime permanent. Le tracé de la courbe d'évolution de $\tau$ en fonction du temps permet néanmoins de vérifier la croissance régulière du coefficient. Si celle-ci n'était pas observée, il serait imprudent de prendre la dernière valeur, qui pourrait s'avérer accidentellement élevée.

\subsubsection{La transmissivité}

L'exemple du chapitre 2 a montré que la transmissivité globale de la fouille, telle que définie en 1.2, était en fait légèrement variable. Ces variations semblent aléatoires (pas de corrélation avec le temps ou un autre paramètre) et sont dues aux incertitudes sur les données mesurées, niveaux et débits. Il est cependant aisé de définir un intervalle d'encadrement de la transmissivité de la fouille, ou encore une valeur moyenne.
Comparons maintenant l'évolution de la transmissivité $T$ et du débit spécifique $\mathrm{Qp} / \mathrm{s}$ en fonction du temps. En prenant l'exemple du chantier d'Axe-Seine, bâtiment E.F.G., pour lequel les mesures de débits sont nombreuses, on obtient la courbe de la figure 9

La courbe de débit spécifique est décroissante en fonction du temps et a pour asymptote la droite $y=T$, ce qui était prévisible compte tenu de l'équation (4) écrite sous la forme:

$$
\frac{\mathrm{Qp}}{\mathrm{s}}=\mathrm{T}+\gamma \cdot \mathrm{S} \cdot \frac{\mathrm{ds}}{\mathrm{dt}}
$$

On remarque qu'à l'échelle des variations de débit spécifique, celles de $T$ sont très faibles: la variation relative $(\Delta T / \Delta(\mathrm{Qp} / \mathrm{s}))$ est en effet de $1,2 \%$.

On peut considérer que la valeur de $\mathrm{T}$ est constante, et ce dès le début du pompage; sur les $40 \mathrm{~h}$, on

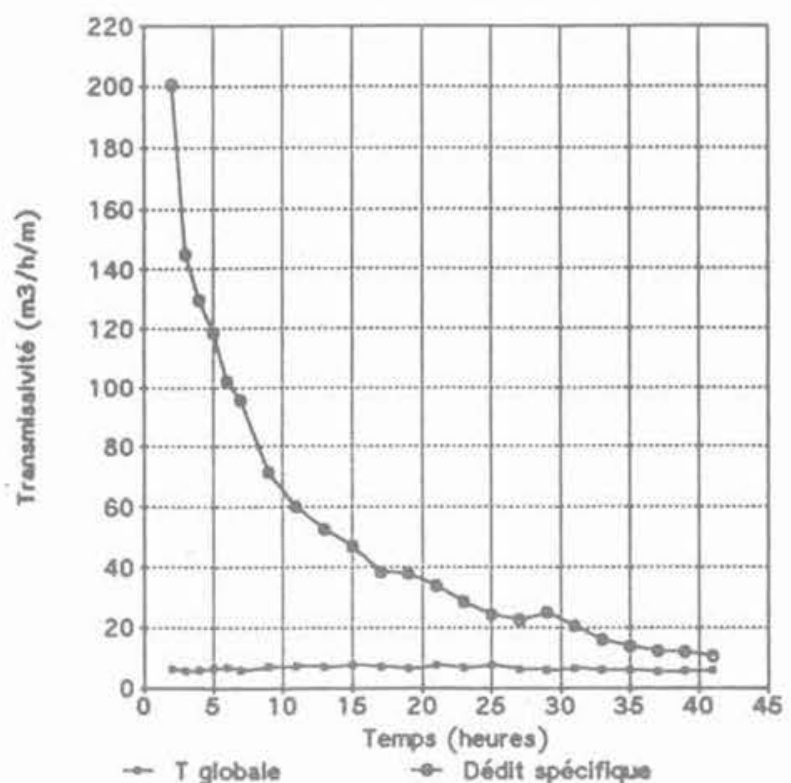

Fig. 9. - Comparaison entre la transmissivité globale et le débit spécifique en fonction du temps.

Fig. 9. - Comparaison between the bulk transmissivity and the specific yield versus time.

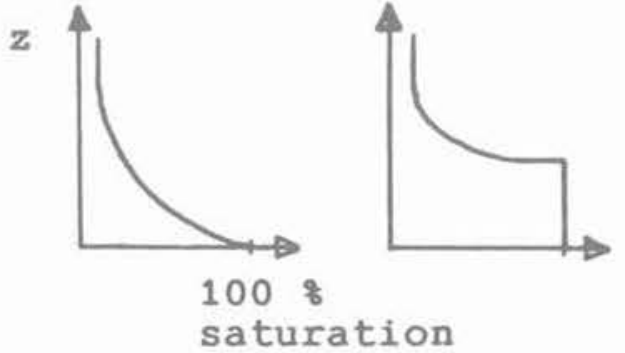

t1
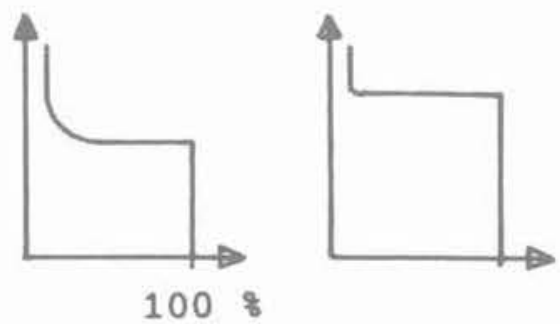

t3

Fig. 8. - Evolution du coefficient de saturation du sol pendant la remontée.

Fig. 8. - Evolution of the water content of the soil during the head recovery. 
calcule une moyenne de $6,6 \mathrm{~m}^{3} / \mathrm{h} / \mathrm{m}$ et un écarttype de 0,6 .

La courbe de débit spécifique ne permet pas une analyse du même type, les valeurs variant très sensiblement en fonction du temps. Au bout de $40 \mathrm{~h}$, la valeur de $\mathrm{Qp} / \mathrm{s}$ obtenue est encore supérieure de $60 \%$ à la moyenne de $\mathrm{T}$.

Cette analyse montre clairement que, même lors d'un essai de pompage où l'on se rapproche de la stabilisation du niveau d'eau intérieur sous pompage, des déductions de débit résiduel faites à partir de la valeur finale de $\mathrm{Qp} / \mathrm{s}$ seraient largement surestimées, et a fortiori lors d'un essai dont la période de pompage s'arrête bien avant la stabilisation.

Une interprétation basée sur un calcul de transmissivité globale permet, au contraire, d'évaluer en quelques heures une valeur quasiment constante.

\subsubsection{Coefficient d'emmagasinement}

II subit le même type de variations que le temps de relaxation car il lui est proportionnel. Ces variations sont de deux types:

\section{- Variations en fonction de la nature du sol}

Il est clair que le coefficient d'emmagasinement varie en fonction du terrain et de son hétérogénéité.

Dans l'essai proposé, aucune hypothèse n'est prise sur ce coefficient. Cependant, les valeurs qui sont déduites de l'interprétation représentent en réalité des valeurs locales de ce coefficient (sur la hauteur de la remontée). L'attribution de ce coefficient à toute la couche dans laquelle s'est déroulé l'essai doit être validée par la connaissance de son homogénéité.

En tout état de cause, il serait très délicat de tirer des conclusions d'un essai dont la remontée s'est effectuée sur deux horizons géologiques différents.

\section{- Evolution en fonction temps}

On mesure le coefficient d'emmagasinement pendant la période de remontée et l'on s'aperçoit qu'il augmente, ce que l'on explique par l'essorage du terrain.

Dans l'exemple du chapitre 2, l'essai se déroulait dans une couche d'alluvions anciennes et l'essorage du terrain était presque terminé au bout de $8 \mathrm{~h}$, le coefficient d'emmagasinement tendant vers une valeur de $16 \%$

Sur un autre chantier présentant les mêmes couches géologiques, la même valeur de $\gamma$ a été retrouvée. Des essais en régime non-équilibré interprétés au moyen de méthodes de type Theis ou Jacob fournissent, d'autre part, dans le cas d'alluvions, des valeurs de coefficient d'emmagasinement comprises entre 10 et $20 \%$ [1].

Par contre, sur les chantiers de l'Aérospatiale à Suresnes ou du nouveau siège de TF1 à Boulogne, les alluvions concernées étaient des alluvions modernes à tendance limoneuse, qui ont montré un essorage beaucoup plus lent. Sur le graphique de l'évolution de $\gamma$ en fonction de la remontée, on a pu observer une courbe à très faible pente ne présentant pas d'asymptote. Les valeurs de fin d'essai du coefficient d'emmagasinement étaient de l'ordre de 3 à $4 \%$; mais, l'essorage n'étant pas terminé, aucune valeur raisonnable du coefficient d'emmagasinement réel ou à long terme de la couche concernée ne pouvait être déduite.

\subsubsection{Analyse qualitative des temps de retard}

Au cours d'un essai, la piézométrie observée présente parfois des irrégularités : si d'une manière générale les courbes de rabattement des piézomètres sont très similaires, certaines ne suivent pas l'allure générale. On dit alors que les piézomètres concernés ont sur les autres un temps de retard, ou temps de réponse.

Ce temps de retard est une formulation simple pour exprimer un phénomène très complexe dans lequel peuvent intervenir de nombreux facteurs que nous allons tenter d'identifier et de classer.

Cette étude permettra, lors d'un essai de pompage, de donner une explication plausible du comportement divergent de certains piézomètres d'une part, et d'autre part de prendre les mesures qui s'imposent au niveau de l'interprétation.

\section{a. Les causes}

On peut en identifier au moins trois, qui s'ajoutent l'une aux autres;

- le retard intrinsèque des piézomètres ; leur nature même implique en effet la nécessité d'une différence de pression entre le piézomètre et la nappe pour que celui-ci réagisse;

- le retard caractéristique des piézomètres, dû au mode d'exécution du forage, aux caractéristiques de la crépine (\% d'ouverture, épaisseur des fentes), à la nature du filtre,...

- le retard du niveau même de l'eau en certains endroits de la fouille, dú à un essorage plus lent (perméabilité plus faible localement, présence d'écrans tels que barrettes, zone éloignée des puits de pompage...).

Cette classification est théorique et il est difficile en pratique de faire la part de chacune des causes possibles.

\section{b. Différents types d'anomalies piézométriques}

- Certains piézomètres sont « bloqués " ; ils ne descendent pas ou presque pas durant la période de pompage.

Il suffit souvent de les souffler pour qu'ils reprennent petit-à-petit (il y a toujours un temps de retard) l'allure générale du rabattement.

- Le niveau d'autres piézomètres reste significativement supérieur au niveau moyen. La période de remontée permet en général d'expliquer leur comportement : le pompage étant arrêté, si le niveau de ces piézomètres continue à descendre, alors que celui de tous les autres remonte, cela signifie qu'ils ont un important temps de retard, qui peut être dâ aux différentes causes exposées en a.

C'est le cas du piézomètre 13 de l'exemple cité qui a continué de baisser alors que le pompage était arrêté ; tant que son niveau était au-dessus du niveau général, il n'a pas rattrapé son retard. Dans ce cas particulier, l'éloignement du piézomètre 13 de la zone de pompage semble être la cause du retard. 


\section{c. Mesures à prendre lors de l'interprétation}

L'interprétation de l'essai étant basée sur lhypothèse d'une nappe plane à l'intérieur de la fouille, l'évaluation de son niveau est représentée par la moyenne des niveaux des piézomètres. Il est clair que la prise en compte des piézomètres retardés a pour effet d'augmenter artificiellement ce niveau moyen, de diminuer le rabattement, et enfin de majorer le débit résiduel. Lorsqu'il apparait que certains piézomètres ne suivent pas l'allure générale en raison d'un important temps de retard, comme c'était le cas du piézomètre $\mathrm{P}_{2} 13$ de l'exemple, il convient de les ignorer dans les calculs d'interprétation, tout en s'assurant qu'ils tendent à rattraper leur retard durant la période de remontée.

\section{d. Effet des temps de retard en phase transitoire}

Le paragraphe précédent règle le problème des piézomètres ayant des temps de retard relatifs importants. Cependant la moyenne des niveaux des piézomètres présente par rapport au niveau réel de la nappe un certain temps de retard, qui n'est pas éliminé dans l'interprétation, notamment da au retard intrinsèque des piézomètres. En régime * permanent », ce temps de retard ne semble pas gênant: il se traduit par un décalage de l'origine des temps, (or l'interprétation est basée sur des calculs par intervalle) et par de faibles variations.

Par contre, en régime transitoire, comme c'est le cas au début de la remontée, deux phénomènes se superposent :

- le temps de retard des piézomètres, qui se transforme en avance, en raison du changement de direction du mouvement du niveau d'eau à l'intérieur de la fouille

- l'essorage du terrain.

Au début de la remontée, le niveau dans le piézomètre est supérieur au niveau de la nappe. En conséquence, malgré l'arrêt du pompage et le fait que la nappe remonte, le piézomètre continue d'exprimer une baisse de niveau. Le niveau du piézomètre passe ensuite par une phase de "stabilisation artificielle, au moment où le niveau d'eau réel et le niveau du piézomètre sont identiques (cela se traduit par un temps de relaxation infini, négatif puis positif)

L'eau continue de monter et le piézomètre reprend son retard.

Puis, le niveau d'eau tend à se stabiliser dans la fouille. La courbe de l'évolution du niveau piézomètre passe donc par un point d'inflexion qui se traduit par un minimum sur la courbe du temps de relaxation.

On arrive alors dans une phase de régime quasipermanent où le temps de retard ne s'exprime plus lors de considérations par intervalles de temps.

Par contre, l'influence de l'essorage, masquée jusqu'alors, subsiste et permet d'expliquer l'augmen- tation régulière du temps de relaxation : l'essorage a pour effet de diminuer artificiellement $\tau$, qui tend vers sa valeur limite à mesure que l'essorage s'atténue.

Ces considérations permettent d'expliquer les variations de forte amplitude du début de remontée.

D'autre part, la durée de ces variations est aussi une borne supérieure du temps de retard global des piézomètres ( $3 \mathrm{~h}$ dans le cas de l'exemple, et entre 2 et $4 \mathrm{~h}$ d'une façon générale).

Une simulation a été faite en prenant l'hypothèse d'un temps de retard global des piézomètres de $30 \mathrm{~min}$, et une même forme de courbe que celle de l'exemple a été obtenue, confirmant l'analyse précédente du phénomène.

Dès lors il est important, lors de l'essai de pompage, de prolonger les mesures de la remontée après l'observation de pics, qui peuvent représenter une surestimation de $\tau$ et ainsi conduire à une sous-estimation du débit résiduel.

Pour la suite, l'interprétation se base naturellement sur la dernière valeur de $\tau$ obtenue (voir explication en 2.).

\section{CONCLUSION}

La méthode d'essai et d'interprétation décrite dans cet article répond au besoin nouveau d'évaluer un débit résiduel dans une enceinte fermée, ce que les essais classiques de pompage, adaptés aux espaces semiinfinis, n'étaient pas en mesure de fournir.

La méthode proposée, validée par la vérification d'une hypothèse fondamentale : la constance de la transmissivité de la fouille, a en outre pu être testée sur divers chantiers, où elle s'est avérée très satisfaisante, tant à court qu'à plus long terme.

Il est cependant important de rappeler que la précision de cette méthode est tributaire de celle des mesures faites sur le chantier, et que ces dernières doivent avant toute interprétation être examinées avec attention.

D'autre part, lors du début de la seconde partie de l'essai, c'est-à-dire la remontée, on observe durant quelques heures des phénomènes transitoires qui ne sont pas pris en compte dans le modèle. Il est donc indispensable de poursuivre l'essai au-delà de cette période.

Enfin, l'essai proposé est pleinement compatible avec les conditions de chantier, et présente l'avantage d'être précis et rapide.

\section{BIBLIOGRAPHIE}

[1] CASTAGNY G. (1963), Traité pratique des eaux souterraines, Dunod.

[2] MAYER A. (1954), Les terrains perméables, Dunod. 